\title{
Charge transport in metal/semiconductor/metal devices based on organic semiconductors with an exponential density of states
}

Citation for published version (APA):

Ramachandhran, B., Huizing, H. G. A., \& Coehoorn, R. (2006). Charge transport in metal/semiconductor/metal devices based on organic semiconductors with an exponential density of states. Physical Review B, 73(23), 233306-1/4. [233306]. https://doi.org/10.1103/PhysRevB.73.233306

DOI:

10.1103/PhysRevB.73.233306

Document status and date:

Published: 01/01/2006

Document Version:

Publisher's PDF, also known as Version of Record (includes final page, issue and volume numbers)

Please check the document version of this publication:

- A submitted manuscript is the version of the article upon submission and before peer-review. There can be important differences between the submitted version and the official published version of record. People interested in the research are advised to contact the author for the final version of the publication, or visit the $\mathrm{DOI}$ to the publisher's website.

- The final author version and the galley proof are versions of the publication after peer review.

- The final published version features the final layout of the paper including the volume, issue and page numbers.

Link to publication

\footnotetext{
General rights

- You may freely distribute the URL identifying the publication in the public portal. follow below link for the End User Agreement:

www.tue.nl/taverne

Take down policy

If you believe that this document breaches copyright please contact us at:

openaccess@tue.nl

providing details and we will investigate your claim.
}

Copyright and moral rights for the publications made accessible in the public portal are retained by the authors and/or other copyright owners and it is a condition of accessing publications that users recognise and abide by the legal requirements associated with these rights.

- Users may download and print one copy of any publication from the public portal for the purpose of private study or research.

- You may not further distribute the material or use it for any profit-making activity or commercial gain

If the publication is distributed under the terms of Article $25 \mathrm{fa}$ of the Dutch Copyright Act, indicated by the "Taverne" license above, please 


\title{
Charge transport in metal/semiconductor/metal devices based on organic semiconductors with an exponential density of states
}

\author{
B. Ramachandhran, ${ }^{1,2}$ H. G. A. Huizing, ${ }^{1}$ and R. Coehoorn ${ }^{1,3}$ \\ ${ }^{1}$ Philips Research Laboratories, Professor Holstlaan 4, 5656 AA Eindhoven, The Netherlands \\ ${ }^{2}$ Materials in Electrical Engineering and Optics, Hamburg University of Technology (TUHH), Eissendorfer Strasse 38, \\ 21073 Hamburg, Germany \\ ${ }^{3}$ Department of Applied Physics, Eindhoven University of Technology, P.O. Box 513, 5600 MB Eindhoven, The Netherlands
}

(Received 1 March 2006; published 16 June 2006)

\begin{abstract}
In amorphous organic semiconductors in which electron or hole transport is due to hopping in an exponential density of states (DOS), the mobility is proportional to $n^{b}$, where $n$ is the carrier density and where $b$ increases with increasing width of the DOS. Exact analytical expressions are given for the steady-state and frequencydependent current density in single-carrier metal/semiconductor/metal devices, based on such materials. For $b \gg 1$, the cross over frequency between a conductive and capacitive ac response is shown to be much larger than the inverse steady-state carrier transit time. The relevance to the analysis of the ac small-signal response in small-molecule and polymer layers, such as are used in organic light-emitting devices, is discussed.
\end{abstract}

DOI: 10.1103/PhysRevB.73.233306

PACS number(s): 72.20.Ee, 72.80.Le

In amorphous organic semiconductors, carrier transport takes place by hopping between energetically disordered localized electron or hole states. The energetic disorder leads to a dependence of the mobility $\mu$ on the carrier density $n$. For the specific case of an exponential density of states (DOS), Vissenberg and Matters ${ }^{1}$ showed that the mobility depends on the carrier density as

$$
\mu=a n^{b}
$$

where $a$ is a material- and temperature $(T)$-dependent prefactor, and where $b=\left(T_{0} / T\right)-1$, with $T_{0}$ the width of the DOS. Equation (1), with $a(T)$ as given in Ref. 1, is valid for small carrier concentrations, and when $T<T_{0}$. A study of the mobility under more general conditions has recently been carried out by Baranovskii et $a .^{2}$ For various organic thin film materials, the field-effect mobility in unipolar and ambipolar organic field effect transistors (OFETs) was found to be consistent with the Vissenberg-Matters model. ${ }^{1,3-5}$ The values found for $T_{0}$, deduced from the transfer curves for OFETs based on pentacene, poly-phenylene-vinylene (PPV), and poly-3-hexyl-thiophene, are typically of the order of 400-550 K. . $^{1,3}$

In this Brief Report, we present exact analytical expressions for the steady-state and ac current density in singlecarrier two-terminal metal/semiconductor/metal devices based on semiconductors that satisfy Eq. (1). These expressions are generalizations for arbitrary values of $b$ of the Mott-Gurney law for the space-charge-limited current (SCLC) under steady-state conditions ${ }^{6}$, and of the expressions for the complex admittance given by Shao and Wright ${ }^{7}$ and Kassing. ${ }^{8}$ Our approach contains several simplifying assumptions, and has to be extended in order to be fully applicable to systems such as organic light-emitting devices, photovoltaic cells, and memories. Nevertheless, the results demonstrate two general consequences of the carrier concentration dependence of the mobility, viz., a modified voltage and layer thickness dependence of the current density, and a critical response frequency (as obtained from the admittance) that is much larger than the inverse of the carrier transit time when $b \gg 1$.

We consider only the drift contribution to the current, and neglect diffusion. The electrodes extend infinitely in the lateral directions. The injecting contact, at $x=0$, is assumed to be Ohmic, and characterized by the carrier density $n(0)$. When $n(0)$ is infinite, the local electric field $E(0)$, vanishes. Realistically, $n(0)$ cannot exceed the density of localized electron or hole sites. Using the Poisson equation $n(x)$ $=(\varepsilon / q) d E / d x$, where $\varepsilon$ is the dielectric constant and $q$ is the elementary charge, the current density is given by

$$
J=q a\left(\frac{\varepsilon}{q} \frac{d E}{d x}\right)^{b+1} E(x) .
$$

Solving this differential equation yields

$$
E(x)=\frac{q}{\varepsilon}\left(\frac{b+2}{b+1}\right)^{(b+1) /(b+2)}\left(\frac{\varepsilon J}{a q^{2}}\right)^{1 /(b+2)}\left(x+x_{0}\right)^{(b+1) /(b+2)},
$$

with

$$
x_{0}=\frac{b+1}{b+2} \frac{\varepsilon J}{a q^{2}[n(0)]^{b+2}} \text {. }
$$

The current is space-charge limited and injection limited for $x_{0} \ll L$ and $x_{0} \gg L$, respectively, where $L$ is the semiconducting layer thickness. In the remainder of this paper, we restrict ourselves to presenting results for the case $x_{0}=0$. The voltage $V$ is obtained by integrating the field over the device thickness. The resulting $J(V)$ relationship is:

$$
J=a \varepsilon\left(\frac{\varepsilon}{q}\right)^{b} \frac{(b+1)^{b+1}(2 b+3)^{b+2}}{(b+2)^{2 b+3}} \frac{V^{b+2}}{L^{2 b+3}},
$$

so that the voltage dependences of $E(x)$ and $n(x)$ are 


$$
E(x)=\frac{2 b+3}{b+2} \frac{V}{L}\left(\frac{x}{L}\right)^{(b+1) /(b+2)}
$$

and

$$
n(x)=\frac{\varepsilon}{q} \frac{(b+1)(2 b+3)}{(b+2)^{2}} \frac{V}{L^{2}}\left(\frac{x}{L}\right)^{-1 /(b+2)} .
$$

The interelectrode transit time is given by

$$
\tau=\int_{0}^{L} \frac{d x}{\mu(x) E(x)}=\frac{(b+2)^{2 b+2}}{(2 b+3)^{b+1}(b+1)^{b+1}} \frac{1}{a}\left(\frac{q}{\varepsilon}\right)^{b} \frac{L^{2 b+2}}{V^{b+1}} .
$$

For $b=0$, i.e., for a constant mobility $\mu=a$, the well-known SCLC result $\tau=(4 / 3) L^{2} /(\mu V)$ is retained. ${ }^{9}$

Equation (5) may be regarded as a generalization of the $J=(9 / 8) \varepsilon \mu V^{2} / L^{3}$ Mott-Gurney law for the SCLC in devices with a constant mobility. ${ }^{6}$ On a double-logarithmic scale the current density varies for $b>0$ still linearly with the voltage, but the slope is larger than 2. Also, the dependence of $J$ on $L$ is stronger. The result is very similar to the expression for the current density across a semiconductor consisting of a host material with a constant mobility $\mu_{h}$, containing trap states with an exponential energy distribution:

$$
J=\mu_{h} \frac{N_{h}}{N_{t r}^{c+1}} \varepsilon\left(\frac{\varepsilon}{q}\right)^{c} \frac{(c+1)^{c+1}(2 c+3)^{c+2}}{(c+2)^{2 c+3}} \frac{V^{c+2}}{L^{2 c+3}},
$$

where $N_{h}$ is the density of host sites, $N_{t r}$ is the density of trap sites, and $c \equiv T_{t r} / T-1$, with $k_{B} T_{t r}$ the width of the trap DOS. ${ }^{10}$ The similarity can be understood as follows. Equation (1) was derived under the assumption that all states are physically equivalent, albeit that there is a distribution of energies. ${ }^{1}$ Therefore, in principle hopping between all states could contribute to the mobility. However, when the carrier density is small and when $T \ll T_{0}$, the mobility is (effectively) due to hopping between scarcely populated states that are situated in an energetically rather narrow zone well above the Fermi level. The peak energy in this zone might be called the "transport energy". ${ }^{2}$ In a simplified picture, only carriers in states at this transport level contribute to the mobility. Therefore, the transport process is, effectively, very similar to that in the case of the exponential trap distribution model, where a distinction between trap states and transport states was already introduced as a starting assumption. It follows that a distinction between both transport modes can only be made on the basis of measurements of the $T$ dependence of the current density, which in Eqs. (5) and (9) is determined by $a(T)$ (see Ref. 1) and $\mu_{h}(T)$, respectively.

Next, we derive the voltage response to a small sinusoidally oscillating current, with frequency $f$ (and angular frequency $\omega=2 \pi f)$, on top of a dc bias current. We use the small-signal approximation, and write the current density, voltage, conductivity, and electric field as sums of steadystate and time-dependent contributions: $J(t)=J_{d c}+j(t), V(t)$ $=\mathrm{V}_{d c}+V(t), \quad \Sigma(x, t)=\Sigma_{d c}(x)+\sigma(x, t), \quad$ and $\quad E(x, t)=E_{d c}(x)$ $+e(x, t), \quad$ respectively, with $j(t)=j^{*} \exp (i \omega t), \quad V(t)$ $=V^{*}(\omega) \exp (i \omega t)$, and $e(x, t)=e^{*}(x, \omega) \exp (i \omega t)$.

The current density is the sum of a drift and a displace- ment term, $J(t)=\Sigma(x, t) E(x, t)+\varepsilon \partial E(x, t) / \partial t$, where the position- and time-dependent effective conductivity is $\Sigma(x, t)=\mathrm{qn}(x, t) \mu(x, t)=\mathrm{qa}[n(x, t)]^{b+1}$. Within the smallsignal approximation, terms that contain products of timedependent functions can be neglected, so that $j(t)$ is given by

$$
\begin{aligned}
j(t)= & q n_{d c}(x) \mu_{d c}(x) e(x, t)+\varepsilon(b+1) \mu_{d c}(x) E_{d c}(x) \frac{\partial e(x, t)}{\partial x} \\
& +\varepsilon \frac{\partial e(x, t)}{\partial t} .
\end{aligned}
$$

The first and third terms are the ac current density due to the ac field and the ac displacement current density, respectively. The second term arises from the time-dependence of the local conductivity. It is derived as follows:

$$
\begin{aligned}
j_{2}(x, t) & =\sigma(x, t) E_{d c}(x) \\
& =q a\left[n(x, t)^{b+1}-n_{d c}(x)^{b+1}\right] E_{d c}(x) \\
& =q a(b+1) n_{d c}(x)^{b}\left[n(x, t)-n_{d c}(x)\right] E_{d c}(x) \\
& =\varepsilon(b+1) \mu_{d c}(x) E_{d c}(x) \frac{\partial e(x, t)}{\partial x} .
\end{aligned}
$$

The second and third steps have been made using the smallsignal assumption and the Poisson equation, respectively. Using Eqs. (1) and (6)-(8), and writing $\xi \equiv x / L$ and $\Omega \equiv \omega \tau$, Eq. (10) can be rewritten as

$$
\begin{aligned}
j^{*}= & \frac{\varepsilon}{\tau}\left(\xi^{(b+1) /(b+2)} e^{*}(\xi, \Omega)+(b+2) \xi^{1 /(b+2)} \frac{\partial e^{*}(\xi, \Omega)}{\partial \xi}\right. \\
& \left.+i \Omega e^{*}(\xi, \Omega)\right) .
\end{aligned}
$$

The solution for which $e^{*}(0)=0$ for all $\Omega$ [so that $E(0, t)$ $=0$ for all $t]$ is

$$
\begin{aligned}
e^{*}(\xi, \Omega)= & \frac{j^{*} \tau}{\varepsilon} \frac{\exp \left(\frac{-i \Omega \xi^{(b+1) /(b+2)}}{b+1}\right)(1+b)^{1 /(1+b)}}{(-i \Omega)^{(b+2) /(b+1)} \xi^{1 /(b+2)}}\left[\Gamma\left(\frac{b+2}{b+1}\right)\right. \\
& \left.-\Gamma\left(\frac{b+2}{b+1}, \frac{-i \Omega \xi^{(b+1) /(b+2)}}{b+1}\right)\right] \equiv \frac{j^{*} \tau}{\varepsilon} \eta^{*}(\xi, \Omega) .
\end{aligned}
$$

The second step defines the dimensionless function $\eta^{*}(\xi, \Omega)$. $\Gamma(a) \equiv \Gamma(a, 0)$, and $\Gamma(a, z)$ are the Gamma function and the incomplete Gamma function, respectively. The complex ac voltage $V^{*}(\Omega)$ is obtained by integrating $e^{*}$ over the device thickness:

$$
V^{*}(\Omega)=\frac{j^{*} \tau L}{\varepsilon} \int_{0}^{1} \eta^{*}(\xi, \Omega) d \xi \equiv \frac{j^{*} \tau L}{\varepsilon} v^{*}(\Omega) .
$$

The last step defines the dimensionless voltage function $v^{*}(\Omega)$. The ac response can be expressed in terms of the complex admittance, defined as $Y(\omega) \equiv A j^{*}(\omega) / V^{*}(\omega)$ $\equiv G(\omega)+i \omega C(\omega)$, with $A$ the device area. $G$ is the differential conductance, 


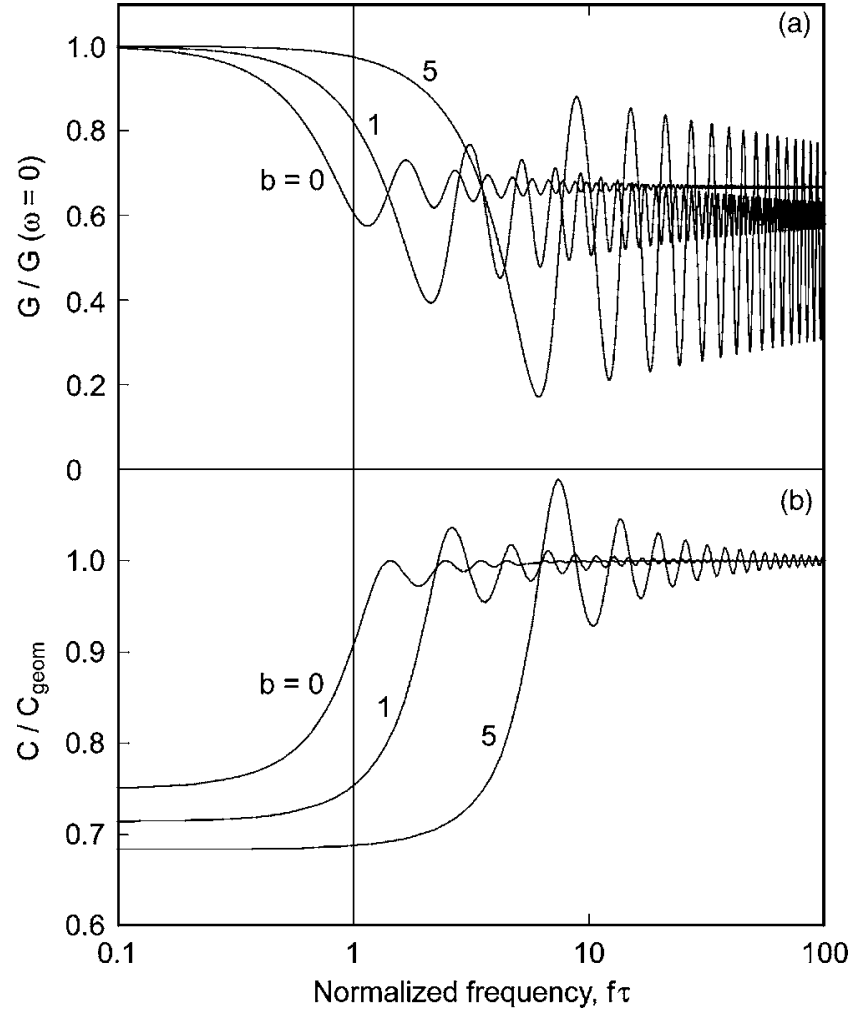

FIG. 1. Normalized differential conductance (a) and capacitance (b) of metal/semiconductor/metal devices, as a function of the normalized frequency, for semiconductors with a mobility given by Eq. (1), with the exponent $b=0,1$, and 5. Ideal Ohmic contacts have been assumed, and diffusion is neglected. $C_{\text {geom }}$ is the geometric capacitance, $f$ is the frequency, and $\tau$ is the carrier transit time [Eq. (8)].

$$
G(\Omega)=\operatorname{Re}\left(\frac{1}{v^{*}(\Omega)}\right) \frac{\varepsilon A}{\tau L}=\operatorname{Re}\left(\frac{1}{(2 b+3) v^{*}(\Omega)}\right) A \frac{d J_{d c}}{d V},
$$

and $C$ is the differential capacitance:

$$
C(\Omega)=\operatorname{Im}\left(\frac{1}{\Omega v^{*}(\Omega)}\right) C_{g e o m},
$$

where $C_{\text {geom }}=\varepsilon A / L$ is the geometric capacitance. Using Eq. (5), $G$ has been expressed in terms of $G(0)=\mathrm{Ad} J_{d c} / \mathrm{dV}$. For the general case $b>0$ and $\Omega>0$, we have not been able to evaluate the integrals $v^{*}(\Omega)$ analytically. We have calculated $v^{*}(\Omega)$ numerically using the mathematical software package MATHEMATICA.

Figures 1(a) and 1(b) show the dependence of the differential conductance and capacitance, respectively, on the normalized frequency, for various values of $b$. In the zerofrequency limit, the capacitance is given by

$$
C(0)=\frac{2 b+3}{3 b+4} C_{g e o m} .
$$

For $b=0, C(0)=(3 / 4) C_{\text {geom }}$, as is well known from the literature. ${ }^{6,7}$ For all $b, C(0)$ is smaller than $C_{\text {geom }}$. In contrast, the "static capacitance," which is defined as the (dc) charge

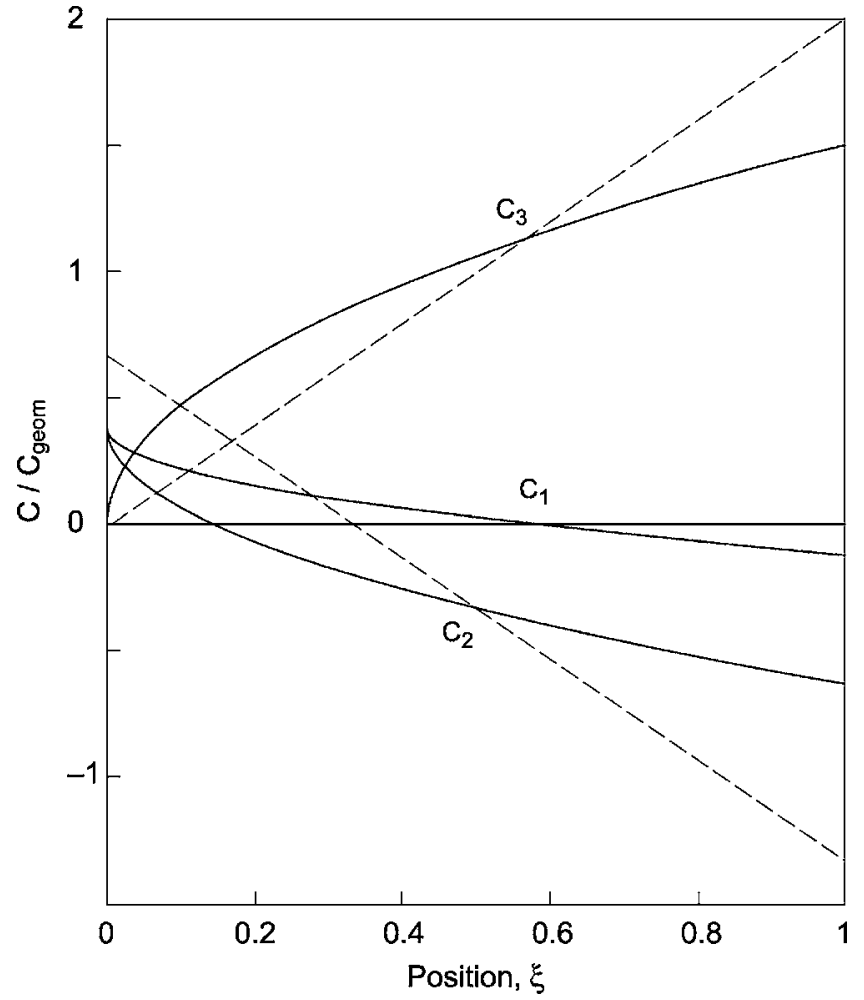

FIG. 2. Normalized contributions $C_{1}, C_{2}$, and $C_{3}$ to the differential capacitance at zero frequency as a function of the dimensionless position in the device, $\xi=x / L$, for semiconductors characterized by mobility exponents $b=0$ (full curves) and $\infty$ (dashed curves). For $b=\infty, C_{1}=0$ for all $\xi$.

density in the semiconductor per unit of (dc) voltage,

$$
C_{s t} \equiv \frac{A}{V} \int_{0}^{L} q n(x) d x=\frac{2 b+3}{b+2} C_{g e o m},
$$

is larger than $C_{\text {geom }}$. For the case $b=0$ the difference between $C(0)$ and $C_{s t}$ has been explained by Kassing, ${ }^{8}$ by expressing $C(\Omega)$ as a sum of three contributions of the form $C_{i}(\xi, \Omega)$ $\equiv \operatorname{Im}\left[j_{i}^{*}(\xi) A \tau / V^{*}(\omega)\right] / \Omega$, with $i=1,2,3$, which correspond to the three contributions $j_{i}$ to the ac current density, given (for any $b$ ) in Eq. (12). $C_{1}$ and $C_{2}$ are due to real particle currents, whereas $C_{3}$ is due to the displacement current. An important point to notice is that the capacitances $C_{i}$ are position dependent. This received little attention in Ref. 8. For the zero-frequency limit, the values for $b=0$ and for $b \rightarrow \infty$ are shown in Fig. 2. At the injecting electrode $C_{1}$ and $C_{2}$ are positive (capacitive), because the particle current is there ahead in phase with respect to the ac voltage: injection becomes progressively more difficult when a space charge builds up. The boundary condition $E(0)=0$ implies that $C_{3}(0)=0$ and that the charge density in the semiconductor induces no image charge at the surface of the injecting electrode. As a consequence, the image charge at the surface of the exit electrode is equal in magnitude to the charge in the semiconductor. That implies that $C_{3}(x=L)$ is equal to the static capacitance. Although $C_{3}(x=L)$ is larger than $C_{\text {geom }}$, the total capacitance is smaller than $C_{\text {geom }}$ because of the 
relatively large negative (inductive) contribution $C_{1}+C_{2}$ due to the time-delayed arrival of carriers at the exit electrode.

Figure 1 reveals a crossover from a low-frequency regime in which $G$ and $C$ are almost independent of $f$ to a highfrequency regime in which both quantities oscillate with $f$. In these regimes $G \gg \omega C$ and $G \ll \omega C$, respectively, and the voltage response to an ac current is almost in phase and out of phase, respectively. The critical response frequency $f_{c r}$ may be defined in several ways. The definition $\left(2 \pi f_{c r}\right) C\left(f_{c r}\right) \equiv \pi G\left(f_{c r}\right)$ leads to $f_{c r}=\tau^{-1}$ for $b=0$, and to $f_{c r}$ $\approx(1+0.65 b) \tau^{-1}$ for arbitrary $b$. Alternatively, defining $f_{c r}$ as the frequency at which a linear extrapolation of the lowfrequency part of the $C(f)$ curve, through the first inflection point, crosses the $C=C_{\text {geom }}$ level leads to $f_{c r} \approx(b+1.25) \tau^{-1}$. Irrespective of the precise definition of $f_{c r}$, an important conclusion is that for $b \gg 1, f_{c r}$ is significantly larger than $\tau^{-1}$. This can be understood from the presence of the factor $(b+1)$ in the second term in Eq. (10). The carrier concentration dependence of the mobility effectively enhances the mobility [see Eq. (11)]. Indeed, we find that for $b \gg 1$ the capacitance, as evaluated at $x=0$, is at any frequency predominantly due to $C_{2}$ (i.e., $C_{2} \gg C_{1}$, whereas $C_{3}=0$ ).

In order to be able to make a full comparison with the experimental admittance for disordered systems, the model should be extended in several directions. First, a deficiency of Eq. (1) is that it is based on the assumption of local thermal equilibrium. It neglects the finite capture and escape times of carriers that hop into and out of deep states, respectively. As shown by Kassing for the case of trap states at a single energy level, ${ }^{8}$ this can lead to a capacitance that increases with decreasing frequency, for $f<f_{c r}$. The capaci- tance shows then a minimum just below $f_{c r}$. Martens et al. ${ }^{11}$ studied the admittance of PPV-based hole-only devices, and observed indeed such a minimum. Second, diffusion cannot always be neglected, e.g., at high frequencies, $G$ and $C$ are predicted to oscillate around $G(\infty)=(b+2) /(2+b+3) G(0)$ and $C_{\text {geom }}$, respectively, with an amplitude that decreases with increasing frequency. In this regime, the ac current density oscillates with a spatial period that is much smaller than $L$. As a result, diffusion is expected to damp the oscillations. Recently, this has indeed been demonstrated by Gommans et $a l .{ }^{12}$ from numerical calculations for the case $b=0$. Also experimentally, no oscillations were observed in the conductance and capacitance for PPV-based hole-only devices. ${ }^{11}$ Third, the shape of the DOS is certainly not always (fully) exponential. For small carrier concentrations the mobility is generally observed to be concentration independent, as described phenomenologically by $\mu=\mu_{0}+a n^{b}, 3$ or as described by expressions that follow for hopping in a Gaussian DOS. ${ }^{13}$

In conclusion, the rather simple form of Eq. (1) has made it possible to obtain analytical expressions for the steadystate current density [Eq. (5)] and the complex admittance [Eq. (15)] for a semiconducting layer with an exponential DOS. The results will provide a useful exact benchmark in future numerical studies that include the effects of a nonequilibrium site occupation and diffusion.

\section{ACKNOWLEDGMENTS}

We thank H. C. F. Martens, S. L. M. van Mensfoort, R. van der Hout, and A. J. E. M. Janssen for their helpful suggestions.
${ }^{1}$ M. C. J. M. Vissenberg and M. Matters, Phys. Rev. B 57, 12964 (1998).

${ }^{2}$ S. D. Baranovskiï, O. Rubel, and P. Thomas, Thin Solid Films 487, 2 (2005).

${ }^{3}$ C. Tanase, E. J. Meijer, P. W. M. Blom, and D. M. de Leeuw, Phys. Rev. Lett. 91, 216601 (2003).

${ }^{4}$ E. Calvetti, L. Colalongo, and Zs. M. Kovacs-Vajna, Solid-State Electron. 49, 567 (2005).

${ }^{5}$ E. C. P. Smits, T. D. Anthopoulos, S. Setayesh, B. de Boer, R. Coehoorn, E. van Veenendaal, P. W. M. Blom, and D. M. de Leeuw, Phys. Rev. B 73, 205316 (2006).

${ }^{6}$ N. F. Mott and R. W. Gurney, Electronic Processes in Ionic Crystals (Clarendon Press, Oxford, 1940).
${ }^{7}$ J. Shao and G. T. Wright, Solid-State Electron. 3, 291 (1961).

${ }^{8}$ R. Kassing, Phys. Status Solidi A 28, 107 (1975).

${ }^{9}$ M. A. Lampert and P. Mark, Current Injection in Solids (Academic, New York, 1970).

${ }^{10}$ P. Mark and W. Helfrich, J. Appl. Phys. 33, 205 (1962).

${ }^{11}$ H. C. F. Martens, H. B. Brom, and P. W. M. Blom, Phys. Rev. B 60, R8489 (1999).

${ }^{12}$ H. H. P. Gommans, M. Kemerink, and W. H. A. Schilders, Phys. Rev. B 72, 165110 (2005).

${ }^{13}$ W. F. Pasveer, J. Cottaar, C. Tanase, R. Coehoorn, P. A. Bobbert, P. W. M. Blom, D. M. de Leeuw, and M. A. J. Michels, Phys. Rev. Lett. 94, 206601 (2005). 